\title{
Remontée d'un anneau de voussoir : modélisation et analyse paramétrique
}

N. ROUSSEL

Laboratoire central des ponts et chaussées Division BCC 58, boulevard Lefëbvre 75732 Paris Cedex 15 Nicolas.roussel@lcpc.fr

\section{LANOS}

Z. TOUTOU

Groupe de recherche génie civil Rennes Équipe Matériaux INSA de Rennes, CS 14315 20, av, des Buttesde-Coesmes 35043 Rennes Cedex

\begin{abstract}
Les mortiers de bourrage injectés entre le sol et l'anneau de voussoirs en béton armé d'un tunnel s'apparentent à des fluides à seuils. L'anneau de voussoir peut, en fonction des sollicitations mécaniques qu'il subit, se déplacer au sein de ce fluide. La modélisation d'un tel problème d'écoulement de fluide plastique permet d'estimer les efforts nécessaires à la mise en mouvement du tunnel dans une direction quelconque ou d'évaluer le risque de remontée d'un anneau de voussoir soumis à un effort de type "poussée d'Archimède ». A cette fin, un outil de calcul analytique est développé. L'incidence des paramètres géométriques, rhéologiques et tribologiques sur les risques de mouvement de l'anneau est alors étudiée. Les résultats obtenus sont comparés à ceux provenant d'un calcul par éléments finis.
\end{abstract}

Mots-clés : écoulement plastique, mortier, tunnel, poussée d'Archimède.
NDLR: Les discussions sur cet article sont acceptées jusqu'au

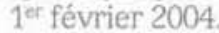

\section{Concrete tunnel ring movement risk: modeling and parametric analysis}

The mortars injected between the ground and the reinforced concrete segment lining of a tunnel are considered as fluids with threshold. The tunnel concrete ring can, according to the mechanical loading which it undergoes, move within this fluid. The modeling of such a plastic fluid flow problem makes it possible to evaluate the efforts necessary to the actuation of the tunnel in an unspecified direction or to evaluate the ring movement risk due to the "Archimede effect $)$. An analytical computational tool is developed. The analysis of the geometrical. rheological and tribological parameters on the ring movement risks is then studied. The obtained results are compared with those coming from a calculation using finite element method.

Key words : plastic flow, mortar, tunnel, buoyant force. 


\section{Introduction}

Lors de la réalisation d'un tunnel à l'aide d'un tunnelier, le tube en béton armé de l'ouvrage est réalisé par la pose successive d'anneaux de voussoirs. Un mortier de bourrage est injecté entre le sol et ces anneaux. Les mortiers utilisés sont souvent composés de sable, de cendres volantes, de laitiers, éventuellement additionnés de ciment ou de bentonite. Après son injection et pendant une période variable, le mortier s'apparente à un fluide à seuil.

Un anneau de voussoir peut, en fonction des sollicitations mécaniques qu'il subit, se déplacer au sein du mortier de bourrage. Ainsi, la remontée des anneaux d'un tunnel est un phénomène couramment rencontré quí est, dans l'état actuel des connaissances, essentiellement attribué à la «Poussée d'Archimède », c'est-àdire la différence de densité entre le mortier de bourrage et le tunnel. La remontée est conditionnée par les résistances à l'écoulement opposées par le mortier.

Les autres origines possibles du mouvement sont les courbures du tracé du tunnel, aussi bien dans le plan vertical que dans le plan horizontal, les efforts de vérinage nécessaires à la progression du tunnelier associés à l'excavation par la roue de coupe. Enfin, les différentes phases de creusement, amenée des voussoirs, pose des voussoirs et injection, peuvent également entraîner de grandes variations du poids volumique équivalent du tunnel induisant des "poussées d'Archimèden très variables au cours d'une séquence de creusement. De tels déplacements du tunnel peuvent générer de forts surcoûts pour ne pas nuire au fonctionnement en service de l'ouvrage.

L'objectif de l'étude est d'analyser les conditions de ce mouvement dans le mortier de bourrage. Les travaux présentés ont été réalisés dans le cadre du Projet national Eupalinos (2001). La modélisation adoptée pour étudier le problème tient compte de différents paramètres géométriques (diamètre du tunnel, épaisseur de la zone injectée, excentrement initial de l'anneau de voussoir), rhéologiques (loi de comportement du mortier frais) et tribologiques (caractérisation mécanique des interfaces mortiertunnel, et mortier-sol). Le comportement rhéologique associé aux mortiers correspond à de la plasticité parfaite. Les conditions de frottement aux interfaces sont choisies de type seuil. La modélisation est bidimensionnelle et sa résolution reste analytique. L'équilibre d'une couronne de mortier est construit selon le principe de l'analyse limite. Cette analyse est menée quel que soit le positionnement initial du tunnel dans l'anneau de mortier et quelle que soit la direction du mouvement associé au tunnel.

Le calcul des efforts nécessaires à la mise en mouvement dans une direction quelconque ou l'évaluation du risque de remontée d'un anneau de voussoir soumis à un effort de type "poussée d'Archimèden sont envisagés. Les résultats obtenus sont comparés à ceux provenant d'un calcul par éléments finis.

\section{2}

\section{Rhéologie et tribologie du mortier frais}

Des essais au scissomètre sur différentes compositions de mortiers de bourrage ont été effectués dans le cadre du Projet national Eupalinos. Les résultats obtenus permettent d'associer aux mortiers testés un caractère essentiellement plastique en particulier sous faibles vitesses de cisaillement, ce qui est le cas dans l'étude de la remontée d'anneau. Les valeurs caractéristiques des seuils de plasticité ont été identifiées. Ces seuils sont voisins de $2 \mathrm{kPa}$ pour un mortier frais ( $30 \mathrm{mn}$ après sa préparation) et voisins de 6 à $7 \mathrm{kPa}$ après $24 \mathrm{~h}$. Il a été montré, par ailleurs, que le matériau ainsi préparé peut être considéré comme incompressible.

Des essais réalisés à l'aide d'un tribomètre (appareil permettant d'évaluer les conditions de frottement d'un échantillon de mortier sur une plaque métallique (Djelal, 1991)) permettent d'associer un comportement tribologique de type Coulomb à l'interface sur une plage de vitesses de 0 à $20 \mathrm{~mm} / \mathrm{s}$. Ces résultats sont obtenus sans maitrise des conditions de drainage de l'échantillon. En conditions non drainées, les cisaillements aux interfaces (mortier-sol et mortier-voussoir) sont considérés de type seuil. Ces seuils peuvent atteindre $50 \%$ du seuil de plasticité et sont fortement dépendants de la rugosité à l'interface.

\section{3}

\section{Modélisation}

\section{1}

\section{Géométrie étudiée et hypothèses de calcul}

Le diamètre d'un anneau de voussoirs peut varier de 2 à 15 m environ. Les épaisseurs moyennes de mortier de bourrage injecté derrière ces voussoirs varient entre 10 et $20 \mathrm{~cm}$. De telles conditions géométriques permettent de supposer que, durant un mouvement du tunnel, tout point du mortier est en écoulement. En général, les mouvements constatés pour un tunnel sont insuffisants pour entraîner le contact entre l'anneau de voussoirs et le sol.

La modélisation du problème repose sur une discrétisation radiale de la couronne de mortier (adaptation de la méthode des tranches). Pour chaque tranche ainsi construite, les équations d'équilibres sont établies en considérant que :

- les effets de bords dans la direction de l'axe du cylindre formé par l'anneau de voussoirs sont négligés et on se limite à un problème en deux dimensions traité dans le plan de la figure 1 ;
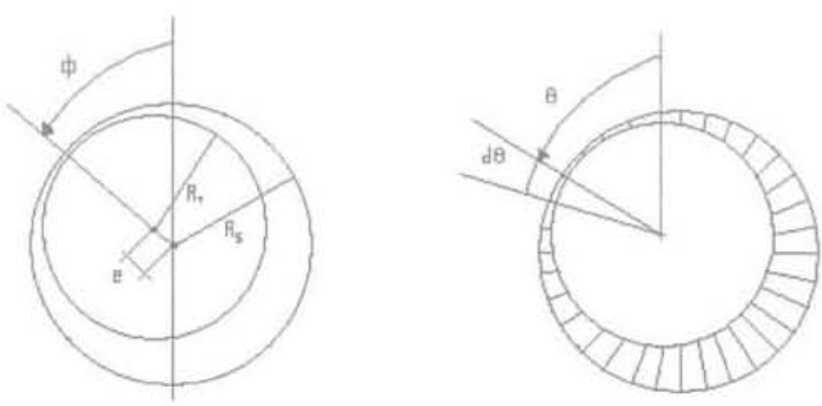

FIG.1 Géométrie du problème et discrétisation. Problem geometry and discrete decomposition. 
- le comportement plastique du mortier est modélisé à l'aide d'un critère de plasticité de type Von Mises (1928). Le mortier est en écoulement plastique en tout point ;

- les cisaillements aux interfaces (mortier-sol et mortier-voussoir) sont de type seuil ;

- l'orientation des contraintes de cisaillement aux interfaces est imposée par le sens de l'écoulement du mortier autour des voussoirs et donc par la direction du mouvement du tunnel étudiée :

- l'effet de la gravité sur le mortier de bourrage est négligé par rapport aux autres efforts mis en jeu.

\section{2}

\section{Construction de la modélisation}

L'anneau formé par les voussoirs cylindriques de rayon $R$, est mis en place dans une excavation cylindrique de rayon $R_{s}$ avec une excentricité e dans une direction formant un angle $\phi$ avec la verticale (v. Fig. 1). Les axes des deux cylindres sont considérés comme parallèles.

L'espace entre les voussoirs et le sol est comblé par le mortier de bourrage de seuil de plasticité $k$ au sens du critère de Von Mises. La contrainte de cisaillement mobilisable à l'interface voussoir-mortier est notée $k_{\text {um }}$ et celle à l'interface sol-mortier est notée $k_{\mathrm{sm}}$.

D'un point de vue mécanique, en traitant le problème dans un repère cylindrique $(r, \theta)$, si le voussoir se déplace à l'intérieur de l'espace disponible, un champ de pression $p$ est amené à se développer. Ce champ de pression est associé à un champ de contraintes déviatoriques $\sigma_{0}$ (contrainte normale) et $\tau$ (contrainte de cisaillement). Les grandeurs $p, \sigma_{\theta}$ et $\tau$ sont considérées comme des fonctions de $\theta$ homogènes sur toute l'épaisseur (dist ${ }_{\mathrm{B}}$ ) du mortier de bourrage.

L'étude va être menée en écrivant l'équilibre successif de tranches de matériau (v. Figs. 1 et 2). Les paramètres $\alpha$, d $\alpha$, dist $t_{\alpha}$ dist $t_{\theta-\alpha e}$ sont calculés à partir des données géométriques du problème et de $\theta$ et d $\theta$.

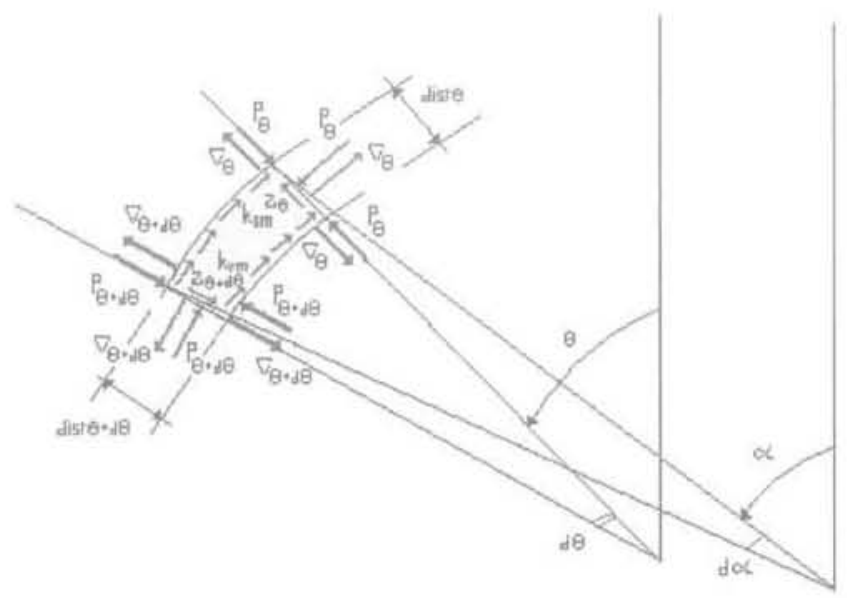

FIG. 2 Discrétisation radiale et équilibre de la couronne de mortier.

Radial discrete decomposition and equilibrium of the studied mortar volume element.

\section{Équations d'équilibre}

Les deux équations d'équilibre en force et l'équation d'équilibre en moment sont écrites pour chaque tranche. Dans le cadre d'une analyse limitée au premier ordre, on peut considérer que les projections dans les directions radiales et orthoradiales des contraintes appliquées sur les surfaces cylindriques sont calculées en considérant :

$$
R_{s} d \alpha=\left(R_{i}+\text { dist }_{\theta}\right) d \theta
$$

Le critère de Von Mises doit être respecté :

$$
\frac{\sigma_{\theta}^{2}}{2}+\tau_{\theta}^{2}=k^{2}
$$

L'hypothèse d'un champ de contraintes homogènes permet de simplifier la résolution du problème mais ne permet pas l'idientification de la solution exacte du système d'équations, On retient finalement les relations de calcul suivantes :

$$
\begin{gathered}
\tau_{\theta}=-\frac{\left(k_{\mathrm{sm}}-k_{\mathrm{sm}}\left(1+\frac{\text { dist }_{\theta}}{R_{1}}\right)\right)}{2} \\
\sigma_{\theta}=\sqrt{2 k^{2}-2 \tau_{\theta}^{2}} \\
d p_{\theta}=\frac{\left(d\left(\text { dist }_{\theta}\right) \sigma_{\theta}-\text { dist }_{\theta} \sigma_{\theta-\mathrm{si \theta}}-k_{\mathrm{vm}} R d \theta\right)-\left(R_{i}+\text { dist }_{\theta}\right) k_{\mathrm{sm}} d \theta}{\operatorname{dist}_{\theta}}
\end{gathered}
$$

\section{4}

\section{Intégrations et conditions aux limites}

De tranche en tranche, les champs de pression et de contrainte peuvent ètre calculés. Une direction servant de condition initiale d'un point de vue mécanique doit cependant être choisie. Cette direction est la direction de déplacement de l'anneau formant un angle $\psi$ avec la verticale. En ce point de discontinuité, les contraintes de friction à l'interface mortier-voussoir changent de sens. L'évolution de la pression est calculée sur l'anneau en analysant sa distribution dans le sens trigonométrique et dans le sens anti-trigonométrique. Le point A (Fig. 3) est identifié à une constante près. En ce point, une égalité des pressions déterminées selon les deux schémas de calcul est obtenue. Ce point correspond ainsi à un minimum de pression. Les valeurs de pression sont donc ajustées de façon à faire apparaitre une pression nulle en A (pression de référence nulle). Notons que, en A, les efforts de friction à l'interface mortier-voussoir changent également de sens. Enfin, ce point A n'est pas systématiquement porté par la direction $180^{\circ}+\psi$ (exemple donné sur la figure 4).

La connaissance du champ de pression sur l'anneau et de la distribution du champ de cisaillement à l'interface mortier-voussoir permet de calculer les composantes verticales $F$ et horizontales $F_{b}$ des efforts appliqués à l'anneau pour induire son déplacement dans la direction $\psi$ (Fig. 3). Ce calcul peut être répété pour toute valeur de $\psi$. 


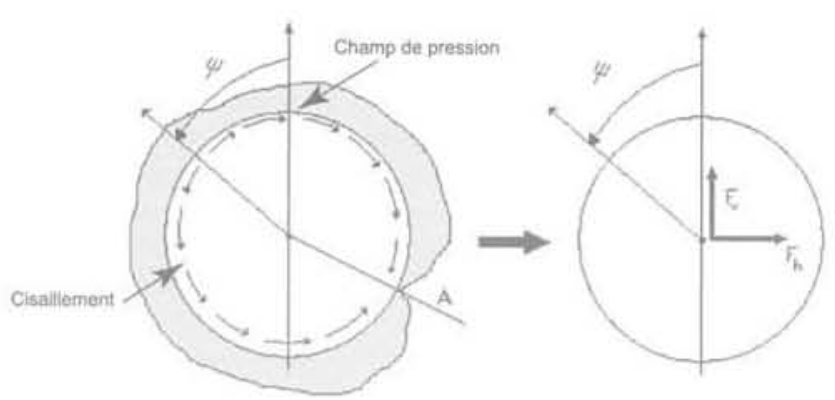

FG.3 Équilibre de l'anneau de voussoirs. Tunnel ring equilibrium.

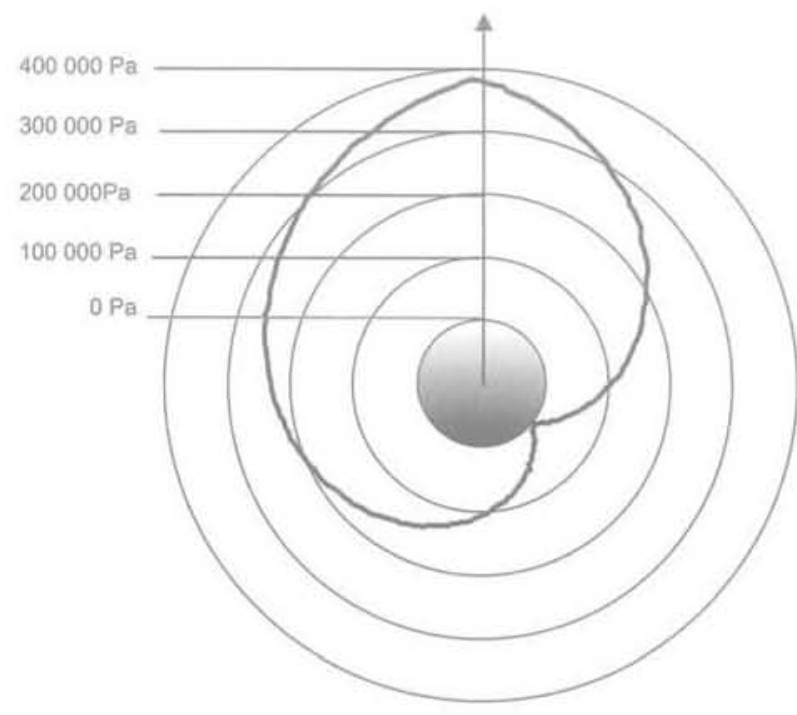

FG. 4 Exemple de répartition de pression. Pressure repartition example.

\section{4}

\section{Construction d'un outil de calcul}

\section{1}

\section{Mise en place du protocole de calcul}

Le système d'équations (3) (4) (5) et le mode de calcul, précédemment construit, ont été implantés dans une feuille de calcul. Les paramètres géométriques et mécaniques du problème sont saisis par l'opérateur. Le calcul direct des efforts $F_{v}$ et $F_{h}$ nécessaires à la mise en mouvement dans la direction étudiée est réalisé. La figure 4 donne un exemple, en coordonnées cylindriques, de l'allure de la répartition de pression obtenue avec l'outil de calcul (zone grisée). L'anneau de voussoir apparait en gris. Sur cette figure l'axe vertical correspond à la direction de déplacement. Cette répartition de pression est obtenue avec les valeurs de paramètre suivantes : $\phi=20^{\circ}, \psi=0^{\circ}$ (déplacement vertical) ;

$k_{\mathrm{vm}}=600 \mathrm{~Pa}, k_{s m}=k=2000 \mathrm{~Pa}$;

$e=0,08 \mathrm{~m}, R_{t}=7,45 \mathrm{~m}$ et $R_{\mathrm{s}}=7,63 \mathrm{~m}$.

\section{Évaluation du risque de remontée de l'anneau de voussoirs}

L'évaluation du risque de remontée du tunnel sous l'effet d'une poussée d'Archimède est analysée en calculant $F$, et $F_{h}$ pour différentes valeurs de l'excentricité $e$, de $\phi$ et de $\psi$.

Pour une configuration initiale du problème, i! existe une unique valeur $\psi_{0}$ pour laquelle $F_{h}=0$. La force s'opposant au déplacement est alors purement verticale. Or la force motrice est la poussée d'Archimède verticale ascendante. C'est donc cette valeur $\psi_{w}$ correspondant à la direction de déplacement de l'anneau pour une configuration initiale donnée, qui est la direction de déplacement privilégiée.

Pour chaque valeur de e et de $\phi$ on détermine donc la direction particulière $\psi_{0}$. L'effort $F$ correspondant est noté $F_{\text {vo }}$. Les calculs sont faits pour des excentricités représentant $0 \%, 33 \%, 50 \%, 67 \%$ et $83 \%$ de $R_{s}-R_{r}$. Les incréments de $\phi$ sont de $10^{\circ}$ et les incréments de $\psi$ de $3^{\circ}$.

Pour évaluer le risque de soulèvement de l'anneau, il convient d'analyser l'équilibre des forces correspondant à la poussée d'Archimède calculée à partir de la masse volumique du mortier, la masse de la structure du tunnel (exprimée pour 1 mètre línéaire) et $F$. La lecture d'un graphique facilite cette évaluation. Différentes interprétations des résultats sont possibles. Par exemple, les évolutions de $F_{v 0}$ ou de $\psi_{0}$ peuvent être analysées en fonction de $\phi$ à $e$ fixé.

La figure 5 illustre l'évolution de $F_{\text {w }}$ en fonction de $\phi$ pour différentes valeurs de $e$. Cette figure est obtenue avec les valeurs de paramètre suivantes:

$k_{\mathrm{vm}}=k_{\mathrm{sm}}=k=80 \mathrm{~Pa}$;

$R_{i}=3 \mathrm{~m}$ et $R_{\mathrm{s}}=3,15 \mathrm{~m}$.

Le poids volumique du mortier est pris égal à $20 \mathrm{kN} / \mathrm{m}^{3}$.

Sur ce graphique l'effort moteur de soulèvement, calculé à partir de la masse volumique du mortier et de la masse linéique du tunnel $(80 \mathrm{t} / \mathrm{ml}$ pour l'exemple de la figure 5), est également tracé. Si les courbes de calcul sont au-delà de cette courbe de référence, quelle que soit la position initiale du tunnel (paramètre e), le soulèvement est impossible. Par contre, si un point d'une des courbes de calcul est en deçà de la courbe de référence, ceci indique qu'il existe certaines positions de tunnel pour lesquelles les risques de déplacement existent. Ainsi, sur la figure 5 la stabilité n'est obtenue que pour des excentricités initiales supérieures à $0,08 \mathrm{~m}$ et cela quel que soit $\phi$.

La lecture directe sur le graphique est possible sans chercher à identifier quelles sont les positions critiques. Cependant, les directions de mouvement intégrées pour un ensemble de positions critiques permettraient de tracer une trajectoire possible du tunnel. Un tel type de traitement des données numériques n'est pas présenté ici, toutefois, les données nécessaires à sa construction peuvent ètre fournies par l'outil de calcul. 


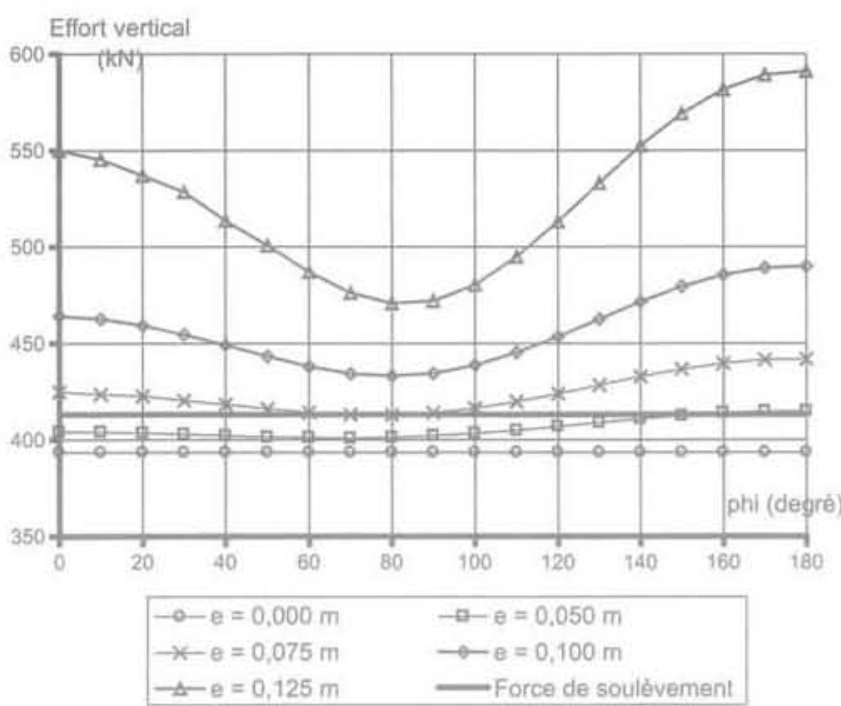

FG, 5 Exemple d'évaluation du risque de remontée.

Tunnel movement risk evaluation example.

\section{5}

\section{Cas limites et analyses paramétriques}

\section{1}

\section{Limites des conditions de modélisation}

Le système d'équations d'équilibre exploité est basé sur la construction d'un champ de contraintes dynamiquement admissible. Ce type de traitement permet de construire une solution du problème qui constitue une borne inférieure de la solution exacte. Ainsi les valeurs d'efforts estimées par l'outil de calcul se placent en sécurité vis-à-vis de l'estimation du risque de remontée du tunnel. Par ailleurs, la simplification de l'écriture des équations d'équilibre reste licite dans la mesure oủ la valeur de $R_{c}-R$, reste faible par rapport à $R_{\text {r }}$ L'épaisseur de la couche de mortier de bourrage doit être suffisamment faible par rapport à la taille du tunnel pour que les hypothèses de champ de contraintes homogènes et d'écoulement généralisé à tout le volume du mortier soient licites.

\section{2}

\section{Analyses paramétriques}

L'outil de calcul peut permettre d'évaluer l'influence de différents paramètres.

L'étude de l'influence d'une excentricité purement verticale sur l'effort vertical nécessaire à un déplacement vertical est réalisée en adoptant les valeurs suivantes pour les paramètres:

$$
\begin{aligned}
& \phi=0 ; \\
& k_{\mathrm{vm}}=k_{\mathrm{sm}}=k=2000 \mathrm{~Pa} ; \\
& R_{\mathrm{t}}=3 \mathrm{~m} \text { et } R_{\mathrm{s}}=3,15 \mathrm{~m} .
\end{aligned}
$$

L'excentricité évolue ainsi de 0 à $14 \mathrm{~cm}$. L'effort nécessaire au déplacement est une fonction croissante de $e$ (Fig. 6). F est minimum si le tunnel est parfaitement centré. Dans cette configuration géométrique, la circulation du mortier de bourrage autour des voussoirs est la plus facile. Plus le tunnel est excentré, plus l'effort nécessaire à son déplacement est important. Ceci explique la stabilisation du phénomène avant que les voussoirs ne touchent les parois de l'excavation sauf dans les cas extrêmes de mortiers très fluides. Si la valeur de l'excentricité initiale tend vers $R_{s}-R_{v}$ la force nécessaire au déplacement tend vers l'infíni, la circulation du mortier de bourrage autour des voussoirs n'étant plus possible.

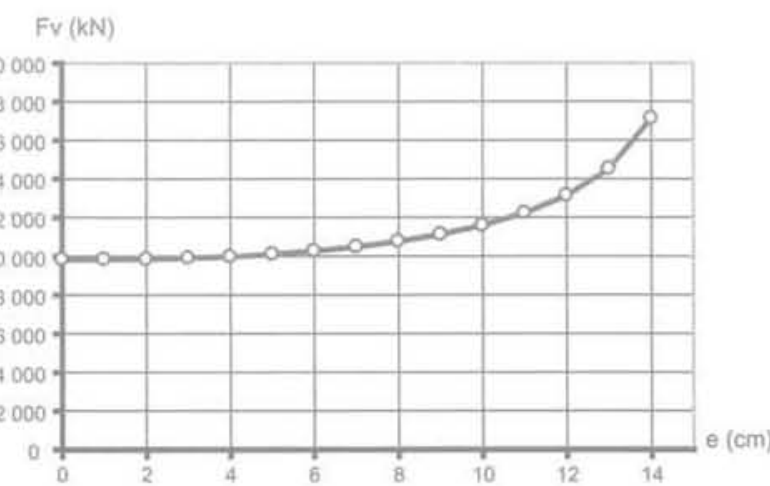

FIG.6 Exemple d'évaluation du risque de remontée.

Vertical force needed to induce a displacement in terms of the initial excentricity.

L'étude de l'influence des conditions de cisaillement aux interfaces est envisagée dans le cas d'un tunnel centré. Les valeurs associées aux paramètres sont :

$$
\begin{aligned}
& \phi=0, e=0 \mathrm{~cm} ; \\
& k=2000 \mathrm{~Pa} ; \\
& R_{t}=3 \mathrm{~m} \text { et } R_{s}=3,15 \mathrm{~m} .
\end{aligned}
$$

$k_{\mathrm{sm}} / k$ évolue de 0 à 1 avec $k_{\mathrm{sm}} / k=1$ ou $k_{\mathrm{str}} / k$ évolue de 0 à 1 avec $k_{\text {vv }} / k=1$. Les résultats sont présentés sur la figure 7 .

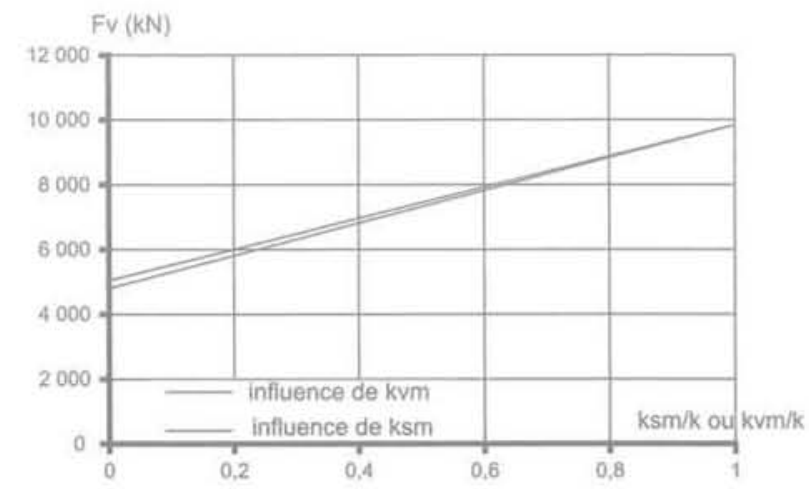

FG.7 Effort vertical nécessaire au soulèvement en fonction des seuils de cisaillement aux différentes interfaces.

Vertical force needed to induce a displacement in terms of the friction yield values at the interfaces. 
Il faut noter que pour un rapport $k_{s m} / k$ ou $k_{y m} / k$ variant de 0 à 1 , l'effort nécessaire au soulevement peut passer du simple au double. La formation d'éventuelles poches d'eau, ou d'autres phénomènes contribuant à abaisser la valeur du seuil de cisaillement de chaque interface, est donc particulièrement à surveiller.

\section{6}

\section{Validation de la méthode de calcul}

La validation de la méthode de calcul proposée repose sur la confrontation des résultats obtenus pour différentes configurations géométriques avec les résultats provenant de calculs numériques exploitant la méthode des éléments finis (simulations réalisées par I'URGC Géotechnique - INSA de Lyon dans le cadre du projet Eupalinos (2001)). Les comparaisons sont limitées à l'estimation du risque de remontée verticale d'un anneau de voussoir excentré verticalement soumis à un effort de type (poussée d'Archimède). Trois exemples d'ouvrages ont été retenus (le tunnel du métro VAL de Rennes, le tunnel du boulevard périphérique Nord de Lyon BPNL et le tunnel de Groene Hart). Les caractéristiques des ouvrages sont indiquées dans le tableau $\mathrm{l}$. Le poids volumique du béton est pris égal à $25 \mathrm{kN} / \mathrm{m}^{3}$. Les valeurs suivantes ont été retenues pour les simulations : $k=2 \mathrm{kPa}, k_{v m}=0,6 \mathrm{kPa}, k_{s y 1}=2 \mathrm{kPa}$. Le poids volumique du mortier est pris égal à $20 \mathrm{kN} / \mathrm{m}^{3}$.

\section{TABLEAU Caractéristiques des tunnels.}

\begin{tabular}{l|c|c|c}
\hline & $\begin{array}{c}\text { VAL } \\
\text { Rennes }\end{array}$ & BPNL & $\begin{array}{c}\text { Groene } \\
\text { Hart }\end{array}$ \\
\hline $\begin{array}{l}\text { Diamètre }(\mathrm{m}) \\
\text { Epaisseur des voussoirs }(\mathrm{m})\end{array}$ & 7,80 & 11 & 14,90 \\
Épaisseur de mortier & 0,34 & 0,45 & 0,60 \\
a injecter $(\mathrm{m})$ & 0,15 & 0,17 & 0,18 \\
Surcharge & - & - & $30 \mathrm{v} \mathrm{mL}$ \\
\hline
\end{tabular}

Pour les simulations numériques aux éléments finis, le module de cisaillement est fixé à $50 \mathrm{kPa}$ et le module de compressibilité à $2,5 \mathrm{MPa}$. Le turnel est considéré centré et subissant un déplacement purement vertical. Le tableau II donne les valeurs de l'effort vertical de soulèvement (poussée d'Archimède, surcharge) appliqué au tunnel, les valeurs de l'effort que le mortier peut opposer au déplacement du tunnel ainsi que le coefficient de sécurité résultant. Un seuil minimum $k$ à donner au mortier pour ne pas avoir de remontée du tunnel est également évalué. Enfin une estimation des déplacements associés à l'obtention de l'effort maximal est donnée.

TABtEau II Résultats des simulations (calculs aux éléments finis).

\begin{tabular}{l|c|c|c}
\hline & $\begin{array}{c}\text { VAL } \\
\text { Fiennes }\end{array}$ & BPNL. & $\begin{array}{c}\text { Groene } \\
\text { Hart }\end{array}$ \\
\hline $\begin{array}{l}\text { Effort de soulèvement (MN) } \\
\text { (effort moteur) }\end{array}$ & 0,7565 & 1,528 & 2,513 \\
$\begin{array}{l}\text { Effort lié à l'écoulement } \\
\text { du mortier (MN) (effort résistant) }\end{array}$ & 1,153 & 2,023 & 3,505 \\
$\begin{array}{l}\text { Coefficient de sécurité } \\
k \text { minimum }(\mathrm{kPa})\end{array}$ & 1,524 & 1,324 & 1,394 \\
Déplacement (mm) & 1,31 & 1,51 & 1,43 \\
\hline
\end{tabular}

Les valeurs reportées dans le tableau III correspondent aux résultats obtenus avec la méthode d'équilibre de tranches. Les valeurs de l'effort vertical de soulèvement appliqué au tunnel sont rappelées. Les valeurs de l'effort que le mortier peut opposer au déplacement du tunnel ainsi que le coefficient de sécurité résultant sont calculées. Un seuil minimal à donner au mortier pour ne pas avoir de remontée du tunnel est également évalué. Afin de comparer ces résultats avec ceux des simulations numériques (tableau II), les calculs sont réalisés avec des excentricités verticales correspondant aux déplacements estimés et présentés dans le tableau II.

TABEAU III Résultats des calculs (méthode des tranches).

\begin{tabular}{l|c|c|c} 
& $\begin{array}{c}\text { VAL } \\
\text { Rennes }\end{array}$ & BPNL & $\begin{array}{c}\text { Groene } \\
\text { Hart }\end{array}$ \\
\hline $\begin{array}{l}\text { Effort de soulèvement (MN) } \\
\text { (effort moteur) }\end{array}$ & 0,7565 & 1,528 & 2,513 \\
$\begin{array}{l}\text { Effort lié à l'écoulement } \\
\text { du mortier (MN) (effort résistant) }\end{array}$ & 1,086 & 1,894 & 3,266 \\
$\begin{array}{l}\text { Coefficient dé sécurité } \\
k \text { minimum (kPa) }\end{array}$ & 1,436 & 1,240 & 1,300 \\
\hline
\end{tabular}

On constate que les résultats des deux simulations sont assez voisins, les écarts restant inférieurs à $7 \%$. La méthode des tranches donne un résultat systématiquement inférieur aux résultats des simulations numériques. Ceci était prévisible puisqu'elle fournit une borne inférieure de la solution exacte.

\section{7 \\ Conclusion}

L'approche selon l'équilibre de tranches, débouche sur la création d'un outil de calcul sans programmation qui constitue un outil d'aide à la conception précieux, simple d'utilisation et rapide. Il permet de peser l'influence des paramètres liés à la géométrie du problème à traiter ainsi que l'influence d'une modification des caractéristiques rhéologiques du mortier.

Les calculs numériques effectués dans cette étude ont permis de mettre en évidence les paramètres clés du phénomène de remontée d'un tunnel au sein de la zone injectée de mortier de bourrage. Outre les caractéristiques géométriques de la zone injectée (épaisseur et diamètre), les caractéristiques de résistance au cisaillement du mortier et de l'interface tunnel-mortier jouent un rôle fondamental. L'effet de l'excentricité initial du tunnel au sein de la zone injectée a également été quantifié.

Il faut cependant noter que la modélisation repose sur des comportements rhéologiques et tribologiques de type seuil dont les valeurs caractéristiques doivent être identifiées. En présence d'un mortier essentiellement visqueux ou viscoplatique, la modélisation devrait être reconsidérée.

Enfin la modélisation est bidimensionnelle. Elle ne peut pas retraduire la possibilité pour le mortier de s'écouler dans la direction axiale du tunnel.

\section{REMERCIEMENTS}

Cette étude a èté financée dans le cadre du Projet national Eupalinos 2000. Elle a été coordonnée par P. Longchamp, Bouygues TP. 


\section{Bibliographie}

Djelal C. - Analyse du phénomène de frottement des mélanges eau-argile concentrés contre des surfaces métalliques. Thèse INSA Rennes, 1991.
Projet national Eupalinos - Rapport de synthèse. Éd. AFTES, 2001.

Von Mises R. - Mechanik der Plastichen Formänderung von Kristallen, Zeitschrift für Amg. Math und Mecchanik, vol. 6, 1928 . 